\title{
Os sistemas de prescrição electrónica
}

André Tomé, ${ }^{*}$ Paula Broeiro, ${ }^{* *}$ António Faria-Vaz ${ }^{* * *}$

\section{RESUMO}

A prescrição electrónica surge da necessidade de melhorar, continuamente, a qualidade dos serviços de saúde prestados e da necessidade de gestão de recursos. Os Sistemas e Tecnologias de Informação da Saúde ou os registos electrónicos em saúde têm evoluído sendo o Reino Unido o melhor exemplo: cerca de $100 \%$ dos médicos, a nível dos cuidados de saúde primários, utilizam, na sua prática diária, os registos médicos electrónicos. Estes dados contrastam com a má adesão a estes sistemas em outros países como os Estados Unidos da América, onde a taxa de utilização ronda os 15\%; em Portugal, a generalização destes sistemas está a ser implementada mas os dados estatísticos são ainda insuficientes.

Os três sistemas utilizados em Portugal, SAM, MedicineOne e VITACARE, permitem a prescrição por Designação Comum Internacional ou por nome de fantasia e as funcionalidades relacionadas com a eficiência administrativa e de gestão de custos são as menos discrepantes. Os grandes atractivos dos sistemas mais evoluídos são o processo centrado no paciente, baseado numa filosofia de trabalho em equipa, do VITACARE e, no MedicineOne, o módulo de prescrição ter sido concebido com o intuito de prestar um apoio clínico efectivo ao prescritor, dispondo por isso de sistemas de alerta funcionais considerados clinicamente relevantes: toxicidade; alertas; interacções. Mesmo a utilização do sistema básico SAM, apesar das suas limitações, é claramente vantajosa em relação à prescrição manual.

As vantagens que se esperam com a adopção de um sistema informatizado de gestão da informação na saúde suplantam os aspectos negativos que se prendem, principalmente, com os apoios informáticos e a vontade profissional de mudar. A eficiência da prescrição electrónica é um dos aspectos mais atractivos. Os aspectos que contribuem para o sucesso da implementação dos sistemas de registo electrónico são: a sua utilidade, a informação neles contida, a utilização amigável e a integração no processo de decisão clínica de normas de orientação clínica em tempo útil.

Palavras-chave: Prescrição Electrónica; Prescrição Automática; Prescrição Computorizada; Eficiência.

A sociedade da informação assenta num modelo de organização e desenvolvimento social e económico onde a informação, como meio de criação de conhecimento, desempenha um papel fundamental na produção de riqueza e na contribuição para o bem-estar e qualidade de vida dos cidadãos. A introdução do registo electrónico na prática clínica é um utilitário de gestão na prestação de cuidados de saúde que permite aumentar a eficiência organizacional e generalizar o acesso e a troca de informação, bem como a sua recolha e análise. $\mathrm{O}$ registo electrónico pode cobrir os diferentes passos do processo da consulta e servir de apoio à decisão e à monitori-

*Interno do Internato Médico de Medicina Geral e Familiar no Centro de Saúde de São Mamede/Santa Isabel

**Assistente Graduada de Clínica Geral no Centro de Saúde do Lumiar

***Chefe de Serviço de Clínica Geral da ARSLVT. Vice-presidente da Comissão

de Ética para a Investigação Clínica (CEIC) zação dos cuidados prestados, quer aos clínicos, quer aos pacientes, quer aos gestores. ${ }^{1}$ Em regra, os sistemas e as tecnologias de informação de apoio à prática clínica incluem os registos dos diários médicos nas suas diferentes modalidades, os pedidos e registos de diferentes intervenções médicas e a prescrição. Apesar do número insuficiente de estudos sobre a avaliação da eficácia destes sistemas, os existentes sugerem que estes sistemas aumentam a segurança dos pacientes e reduzem os gastos, tanto hospitalares, como dos centros de saúde, ou de outras entidades responsáveis pelas despesas de saúde (Quadro I). ${ }^{2,3}$ Num estudo realizado a nível hospitalar, na década de 90, logo no início da introdução do apoio informático, houve interesse em avaliar a sua eficácia. Verificou-se que as equipas da enfermaria hospitalar, que utilizavam os sistemas informáticos, apresentavam menores gastos $(-12,7 \%)$ e uma média de internamento 0,9 dias inferior às das que uti- 
QUADRO I. Vantagens da prescrição electrónica

Na consulta:

- Alertas em tempo real (interacções medicamentosas, ajustes terapêuticos, efeitos adversos, toxicidade)

- Orientações passo-a-passo

- Diminuição de erros na prescrição (alertas em tempo real)

- Abolição de trabalho e/ou registos duplicados

- Melhores registos clínicos (legíveis, completos, fiáveis)

- Diminuição de tempo perdido (por ausência de informação, ou procura da mesma)

- Diminuição do tempo de consulta

- Melhores resultados terapêuticos

Na qualidade dos serviços prestados:

- Estímulo à melhoria da qualidade de serviços

- Possibilidade de avaliar parâmetros de qualidade

- Possibilidade de transmissão de informações directas entre instituições, ou com farmácias

- Abolição de falsificação de receitas

Nos indicadores da instituição:

- Aumento da produtividade e eficiência

- Retorno rápido do investimento

- Diminuição global dos custos em prescrição

- Diminuição dos custos de exames complementares de diagnóstico (pelo registo dos anteriores)

- Diminuição de custos em processos judiciais

lizavam a prescrição manual. ${ }^{4}$ Um outro estudo, de 2003, avaliou o custo-benefício da utilização dos registos electrónicos em cuidados de saúde e chegou a conclusões surpreendentes: apesar dos elevados custos de implementação, suporte técnico, licenciamento, formação e perda de produtividade inicial, ao fim de 5 anos, o investimento foi claramente suplantado pelos lucros conseguidos com a melhoria da gestão do tempo, da informação, dos pedidos de exames evitando duplicações, e da prescrição de fármacos evitando erros. Neste trabalho não foram contabilizados alguns parâmetros, como as vantagens de um esperado aumento da produtividade e da preferência de medicamentos genéricos, ou a poupança conseguida com a diminuição dos casos de má prática, diminuindo custos judiciais e de seguros profissionais, e de incorrecta prescrição medicamentosa, evitando danos nos doentes, ou terapêutica incorrecta com maior consumo de consultas. $^{3}$

A eficiência é assim um dos aspectos mais atractivos: os clínicos do Reino Unido, que utilizam regularmente estes sistemas, afirmam claramente que melhoram os seus índices de produtividade, apesar de nem todos concordarem que reduzem o tempo dispendido pelo clínico. Graças à melhor qualidade de registos dos doentes e/ou aumento da qualidade dos serviços prestados aos doentes crónicos, têm, inclusive, visto os seus rendimentos aumentar, uma vez que são sujeitos a vários parâmetros de qualidade, como ponderação para o cálculo do vencimento. Um outro componente relevante é a eliminação de hiatos de tempo não rentabilizados, duplicação de actos ou registos e o desperdício de tempo porque a informação não está disponível quando é necessária. Alguns investigadores encontraram um desperdício de tempo significativo na procura de informação clínica em $13,6 \%$ das consultas médicas nos cuidados de saúde primários, ${ }^{2}$ sendo um dos principais responsáveis a prescrição manual do receituário. A utilização do sistema electrónico vem acelerar o processo de emissão das receitas, principalmente nos pedidos extensos de medicação prolongada e, inclusivamente, dificultar a falsificação de receitas.

Os erros na prescrição são dos mais frequentes na prática médica, mas também os mais fáceis de evitar. O potencial da prescrição electrónica em reduzir estes erros, devido à ilegibilidade ou falta de dados, é uma das vertentes que mais entusiasmo tem trazido no que respeita aos sistemas de registo e requisição informatizados. Por outro lado, evita que o doente tenha de recorrer ao médico assistente ou não cumprir a terapêutica porque não compreende a prescrição, ou ver recusar a mesma na farmácia por não ser perceptível. Existem mesmo casos que nos permitem compreender a importância dos registos em casos mais delicados, como o caso de um clínico, processado por um doente, que viu as acusações serem invalidadas graças ao registo clínico completo e claro, possibilitado pelo sistema informático que tinha ao seu dispor. A existência de Sistemas e Tecnologias de Informação mais aperfeiçoados permitem integrar e cruzar os vários tipos de informação clínica e de bases de dados (actualizadas regularmente), alertar para as interacções medicamentosas, reduzir a probabilidade de erros na dosagem e adver- 
tir para potenciais reacções tóxicas ou alérgicas. Pode, ainda, disponibilizar formulários ou protocolos prédefinidos, sugerindo fármacos, as suas dosagens, frequência de toma e eventuais alergias, nomeadamente, em relação a medicamentos com os quais o médico tem pouca experiência. Esta integração das várias vertentes possibilita uma melhoria global da qualidade dos serviços prestados (graças aos registos), nomeadamente, pela possibilidade de manter a continuidade dos cuidados prestados. Alguns estudos têm sido realizados no sentido de avaliar se a utilização de Sistemas e Tecnologias de Informação melhoram os resultados dos tratamentos, mas são ainda insuficientes. ${ }^{2,4}$

Até muito recentemente, não se verificou uma aposta política clara nestes sistemas, e as instituições de saúde têm-se debatido com diversos constrangimentos à sua implementação, desde logo os custos da própria instalação (software e hardware), aos custos com a formação e aos decorrentes de uma diminuição da produtividade, resultante da transição entre um sistema de registo manual para um sistema de registo electrónico (Quadro II). ${ }^{5}$ Associado aos problemas anteriormente focados, os próprios profissionais de saúde, nomeadamente os médicos, têm, por vezes, dúvidas quanto ao potencial destes sistemas para evitar os erros e para melhorar a aderência dos doentes à terapêutica. Aliás, a relação médico-doente é por todos assumida como o principal pilar da consulta e da aderência ao tratamento, tanto farmacológico como de outra natureza. De acordo com alguns profissionais de saúde que não são a favor da introdução dos registos electrónicos, a interferência na relação médico-doente é um dos principais constrangimentos à sua plena aplicação, pois a introdução de meios de registo electrónico pode alterar significativamente essa relação. ${ }^{6,7}$

Em Portugal, a oferta de Sistemas e Tecnologias de Informação tem sido uma realidade e é principalmente constituída por aplicações que têm sido desenvolvidas centralmente pelo Ministério da Saúde e por algumas empresas comerciais de informática. Em outros países, o leque de soluções é mais vasto e flexível. .,6,8 $^{-6}$ Nesses, os sistemas informáticos em questão podem ser adquiridos por licença, ou adquiridos gratuitamente no caso de versões básicas. Um aspecto essencial, no que concerne aos sistemas de informação, é a sua compatibilização, de modo a ser possível o cruzamento de
QUADRO II. Limitações à implementação da prescrição electrónica

1. Interferência com a consulta

2. Aprendizagem difícil e demorada

3. Possibilidade de ocorrerem erros de prescrição

4. Impossibilidade de migração dos registos entre sistemas

5. Aumento (receio) do tempo de consulta

6. Dificuldade na actualização regular dos registos

7. Custos elevados de aquisição, manutenção e formação

8. Perda de rendimento durante a adaptação

dados e a mudança de aplicação. Essa impossibilidade poderia implicar a perda dos dados no caso de migração para outro sistema informático, e a impossibilidade de transmissão de dados de saúde, essenciais à monitorização do estado de saúde da população coberta pelos serviços de saúde. ${ }^{4-6,8}$

Em países como os EUA ou o Reino Unido, existe a possibilidade de comunicação bilateral e directa entre o médico prescritor/instituição de saúde e a farmácia, com transmissão das prescrições e indicações para renovação de receituário. Esta capacidade ainda não está desenvolvida em Portugal, mas nos países onde existe funciona como uma mais valia, evitando o extravio ou falsificação de receituário, e sendo mais um factor para melhorar a adesão correcta à medicação. Contudo, muitos dos sistemas informáticos não o permitem, e nem sempre existe feedback positivo das farmácias a esta possibilidade. Inclusivamente, em vários estados dos EUA, a mesma foi impedida, ou suspensa, pela suspeita de ilegalidade na transmissão de informação clínica. ${ }^{6,7}$

Em Portugal, os sistemas electrónicos de registo clínico começam a generalizar-se e, tendo em conta os seus potenciais, procurámos aferir das potencialidades de cada um dos sistemas: SAM (Sistema de apoio ao médico), MedicineOne e VITACARE (Quadros III e IV).

\section{SISTEMA DE APOIO AO MÉDICO}

O SAM inclui um suporte de apoio à prescrição que foi desenvolvido a pensar em questões económicas e não clínicas. Tem um grande potencial de melhoria e, apesar de algumas limitações, a sua utilização é claramente vantajosa em relação à prescrição manual. 
QUADRO III. Suporte à prescrição racional e minimização do risco

\begin{tabular}{|c|c|c|c|}
\hline Funcionalidades & SAM & MedicineOne & VITACARE \\
\hline Alertas de interacções medicamentosas & Insuficiente & + & + \\
\hline Alerta de Insuficiência Renal & Incluída no conjunto de alertas & + & $\begin{array}{l}\text { Não específico da } \\
\text { prescrição }\end{array}$ \\
\hline Alerta de Insuficiência Hepática & Incluída no conjunto de alertas & + & $\begin{array}{l}\text { Não específico da } \\
\text { prescrição }\end{array}$ \\
\hline Alertas de interacções com problemas de saúde & Incluída no conjunto de alertas & + & $\begin{array}{l}\text { Não específico da } \\
\text { prescrição }\end{array}$ \\
\hline Alertas personalizados & - & + & + \\
\hline $\begin{array}{l}\text { Interacções com alimentos, bebidas, } \\
\text { fitoterapêuticos e outras }\end{array}$ & - & + & - \\
\hline $\begin{array}{l}\text { Informação sobre utilização em grupos especiais } \\
\text { (crianças, idosos, amamentação, grávidas) }\end{array}$ & + & - & - \\
\hline
\end{tabular}

\section{QUADRO IV. Eficiência administrativa e gestão de custos}

\begin{tabular}{l|l|c|c}
\multicolumn{1}{c}{ Funcionalidades } & SAM & MedicineOne & VITACARE \\
\hline Prescrição por DCI & + & + & + \\
\hline Prescrição por nome de fantasia & + & + & + \\
\hline Aviso de medicamentos com preço mais baixo & + & + & + \\
\hline Informação sobre comparticipação & + & + & + \\
\hline Renovação de receituário fácil & + & + & + \\
\hline Anulação de receitas até 30 dias & + & + & + \\
\hline Regimes de comparticipação especial & + & Propõe a mais benéfica para o utente & Fácil gestão \\
\hline Base de dados de medicamentos actualizada pelo INFARMED & + & Actualizada semanalmente & + \\
\hline Receitas renováveis dependentes do registo no INFARMED & + & + & + \\
\hline $\begin{array}{l}\text { Renovação de receituário crónico a partir do módulo } \\
\text { de atendimento administrativo ou a partir do portal }\end{array}$ & - & + & +
\end{tabular}

No SAM, a prescrição pode ser efectuada por Designação Comum Internacional (DCI) ou por nome de fantasia. A prescrição por DCI pode ser confusa, em particular para substâncias activas com elevado número de medicamentos genéricos (ex. sinvastatina).

A base de dados de medicamentos é o registo no INFARMED e a sua actualização nem sempre é funcional, excepto quando o medicamento deixou de ser comercializado, em que o sistema não permite a sua prescrição e alerta para o facto. Um dos problemas é a não distinção entre os medicamentos com Autorização de Introdução no Mercado (AIM) e os realmente comercializados.
Os medicamentos que não existam na base de dados (fitoterapêuticos, dietéticos ou outros) podem ser prescritos «à mão» (texto livre), clicando em «outras prescrições». Os dispositivos médicos, como as tiras, agulhas, lancetas e seringas para a diabetes, estão num grupo à parte, acedido através do botão «diabetes». Neste tipo de produtos, para o mesmo nome comercial existem diferentes códigos de barras, o que apresenta alguns constrangimentos.

\section{Regimes de comparticipação especial}

Existe um formulário para inserir regimes especiais de comparticipação, em que o médico tem de escolher o 
tipo de patologia numa lista de opções. É uma grande vantagem, pois o prescritor não precisa de utilizar a memória. Tem como limitação a incapacidade de seleccionar a portaria mais abrangente ou mais favorável ao utente, caso sofra de mais que um problema de saúde abrangido por comparticipação especial. Por exemplo, se o utente é portador de Lúpus Eritematoso Sistémico (LES) e de Depressão, nos antidepressivos coloca a respectiva portaria e não a do LES.

\section{Renovação e anulação de receituário}

O sistema guarda uma lista de todos os medicamentos que foram prescritos anteriormente sob a forma de lista ordenada por data de prescrição e lista de receitas. A lista de medicação prolongada é muito útil na renovação de receituário.

As receitas anteriores podem ser anuladas por vários motivos até 30 dias após a prescrição.

Uma das limitações do sistema, na prescrição de receitas renováveis, é a dependência do registo no INFARMED, sendo impossível prescrever alguns medicamentos de utilização crónica, como os anti-histamínicos.

\section{Sistema de alertas}

O sistema de alertas mistura medicamentos e problemas de saúde, não sendo possível personalizar. Não parece ser funcional: por exemplo, colocar o alerta alérgico à penicilina não impede a sua prescrição.

Nos alertas de interacção de medicamentos com problemas de saúde faltam, por exemplo, alertas de insuficiência hepática e renal. No caso da insuficiência renal, um sistema mais avançado poderia, inclusivamente, alertar o médico para o ajuste de dose em função da clearance da creatinina (calculada a partir de idade, sexo, peso e creatinina sérica, que são parâmetros que, em princípio, já foram registados)

\section{MEDICINEONE}

O módulo de prescrição de medicamentos do MedicineOne foi concebido com o intuito de prestar um apoio clínico efectivo ao prescritor, sem descurar, no entanto, as preocupações financeiras associadas.

A base de dados de medicamentos integrada no MedicineOne tem características únicas em Portugal pois, além de toda a informação disponibilizada pelo INFARMED, disponibiliza ainda toda a informação comple- mentar do medicamento contida no Simposium Terapêutico, incluindo sistemas de prevenção de erro médico, que verificam interacções, contra-indicações, alergias e outros. A actualização desta base de dados é feita de forma automática semanalmente.

As principais características do sistema de prescrição MedicineOne são as seguintes:

\section{Medicação actual e crónica}

Ao aceder ao módulo de prescrição, o médico tem uma visão geral sobre a medicação crónica instituída e sobre a medicação actual. A partir daqui, pode-se rapidamente emitir uma receita com a medicação crónica, redefinir a medicação crónica ou imprimir uma guia de tratamento.

\section{Base de dados apenas com os produtos em comercialização}

A base de dados do medicamento, incluída no MedicineOne (Simposium Terapêutico), não contém as apresentações dos medicamentos que não estão à venda nas farmácias, evitando assim erros na prescrição e diminuindo a necessidade de interacção com as farmácias locais para resolver eventuais problemas de prescrição de embalagens que não se encontram à venda.

\section{Emissão de alertas}

Ao ser adicionado um medicamento à receita, o MedicineOne desencadeia um conjunto de verificações com vista a apoiar o médico na diminuição do erro médico. As verificações efectuadas são as seguintes:

- Interacções entre o medicamento escolhido e os restantes medicamentos prescritos anteriormente e outros que o utente tome actualmente

- Composição do medicamento para verificar se nela existem substâncias às quais o utente é alérgico

- Possíveis contra-indicações do medicamento para estados fisiopatológicos em que o utente se encontre

- Inexistência de comparticipação do medicamento

- Nível de preço muito elevado

Se forem detectadas situações dentro das acima enumeradas, serão apresentadas ao médico explicações clínicas e conselhos ao prescritor.

\section{Controlo da quantidade de embalagens prescritas}

Com base na posologia definida para o medicamento, 
o MedicineOne calcula, automaticamente, o número de embalagens que deverão ser adquiridas pelo utente. Caso o prescritor altere este valor, será feita uma verificação e, se necessário, serão emitidas mensagens de alerta para a situação detectada.

\section{Informações do medicamento disponibilizadas ao prescritor}

Além das informações básicas constantes na base de dados do INFARMED, o Simposium Terapêutico contido no MedicineOne fornece, ainda, um conjunto rico de informação suplementar. As informações são as seguintes:

- Resumo do medicamento com: Nome, Classe ATC, Classe FHNM, Condições de dispensa na farmácia, Condições de conservação, Titular da AIM, Posologia orientativa, Princípios activos, Excipientes e lista de Embalagens disponíveis no mercado

- Monografia completa do produto, tal como se encontra na publicação em livro do Simposium Terapêutico

- Interacções dos princípios activos do produto, com outros princípios activos. Para cada um deles é indicado o sentido da interacção, o tipo, a importância, a gravidade, a probabilidade de ocorrência, a descrição técnica da mesma e conselhos ao prescritor

- Interacções com medicamentos, bebidas e plantas medicinais

- Interacções com alergias, análises clínicas e desporto de alta competição

- Contra-indicações do medicamento

- Utilização especial para grupos de risco

- Dados do produtor e representante

\section{VITACARE}

A aplicação VITACARE tem, como pressuposto base, o conceito de processo único, ou seja, centrado no utente. Isto significa que todos os registos feitos no processo clínico electrónico, independentemente de quem o fez, estão associados ao doente, com identificação de quem e quando foi feito, permitindo a visualização, em tempo real, da totalidade da informação, tendo como única condicionante o perfil do profissional.

\section{Medicação actual e crónica}

Ao aceder ao módulo de prescrição, o médico tem uma visão geral sobre a medicação crónica instituída e sobre a medicação actual. Pode, então e rapidamente, emitir uma receita com a medicação crónica, redefinir a medicação crónica ou imprimir uma guia de tratamento.

\section{Controlo da quantidade de embalagens prescritas}

Com base na posologia definida para o medicamento, o VITACARE calcula automaticamente o número de embalagens que deverão ser adquiridas pelo utente. Caso o prescritor altere este valor, será feita uma verificação e, se necessário, serão emitidas mensagens de alerta para a situação detectada.

\section{Emissão simplificada de várias receitas}

Numa única acção de prescrição, é possível seleccionar medicamentos para receitas de uma ou 3 vias e é também possível isolar medicamentos para serem incluídos em receita separada. Ao mandar emitir a receita, o VITACARE faz a separação dos medicamentos de forma a respeitar a legislação em vigor e as indicações dadas pelo médico.

\section{Renovação do receituário pelo Portal}

A renovação do receituário crónico pode ser feita através do portal da USF, de forma não presencial pelo doente, definindo este que medicamento precisa, número de embalagens e se renovável. O pedido fica automaticamente agendado para resolução por parte do médico. Após emissão do receituário, o doente recebe um SMS ou correio electrónico informando que já pode levantar as receitas

\section{APRECIAÇÃO GLOBAL}

Os três sistemas permitem prescrição por DCI ou por nome de fantasia, e as funcionalidades relacionadas com a eficiência administrativa e de gestão de custos são as que revelam menos diferenças entre o sistema básico SAM e os sistemas mais evoluídos, MedicineOne e VITACARE (Quadro IV).

As funcionalidades específicas dos sistemas de prescrição electrónica melhoram a segurança e a qualidade da gestão terapêutica, prevenindo a iatrogenia (prevenção quaternária). O sistema ideal integra os dados pessoais do paciente; base de dados de medicamentos actualizada; sistemas de alerta hierarquizados por im- 
portância; interacção com farmácias locais (possibilidade de descontinuação de um tratamento); monitorização da adesão à terapêutica. ${ }^{9}$ Dos três sistemas operativos no Sistema Nacional de Saúde (SNS), o MedicineOne parece ser o que mais se aproxima dos sistemas completos de apoio à prescrição (Quadro III).

Habitualmente, na literatura, é utilizada a expressão registo médico electrónico, estando este conceito a mudar. O termo registo electrónico em saúde reflecte a interacção dos diferentes prestadores de cuidados (médicos, enfermeiros, administrativos, outros) e pressupõe a prestação de cuidados suportada em trabalho em equipa e centrada no paciente. ${ }^{10}$ O VITACARE é o sistema que se baseia nesta filosofia, ficando acessíveis ao médico todos os registos efectuados, mesmo por outros elementos da equipa. OVITACARE, na eficiência administrativa e gestão de custos, é quase sobreponível ao MedicineOne e menos exaustivo que este último na minimização do risco.

Num estudo recente publicado no New England Journal of Medicine, a maioria dos médicos utilizadores de sistemas electrónicos de registo clínico refere um efeito positivo:

- na qualidade das suas decisões, da comunicação (com outros prestadores de cuidados e com os pacientes),

- na renovação de receituário,

- na acessibilidade dos registos

- na evicção do erro. ${ }^{11}$

Para os utilizadores de sistemas básicos, a magnitude do efeito foi menor. ${ }^{11}$ Em Portugal, neste momento, falta uma avaliação da eficiência dos três sistemas utilizados.

\section{O FUTURO DOS SISTEMAS ELECTRÓNICOS DE PRESCRIÇÃO}

Os suportes electrónicos de apoio à decisão clínica na gestão da terapêutica têm-se revelado efectivos na redução de prescrição inapropriada, em particular, os sistemas mais evoluídos com sistemas de alerta funcionais. ${ }^{11}$ Os pontos-chave do sucesso da implementação destes sistemas são: a eficiência, a utilidade, a informação contida, a utilização amigável e a integração no processo de decisão clínica de normas de orientação clínica em tempo útil. ${ }^{12}$ Os sistemas de alerta (Figura 1) considerados como clinicamente relevantes foram: os

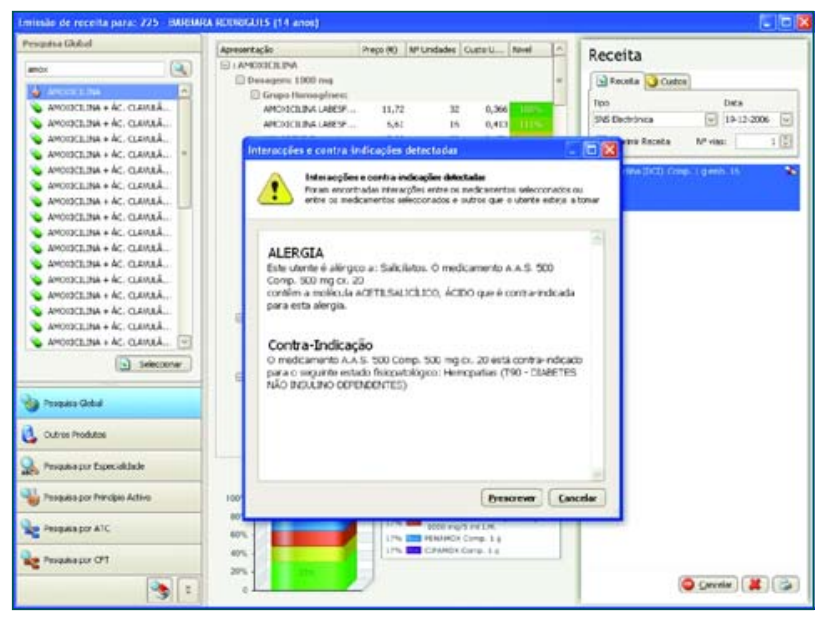

Figura 1. Sistema de alerta activo.

efeitos adversos (toxicidade); as interacções medicamento/doença, medicamento/contra-indicação, medicamento/idade, medicamento/medicamento, medicamento/alergia; ajuste de dose na insuficiência renal ou hepática; duração excessiva do tratamento; duplicações. ${ }^{9,13}$

As vantagens que se esperam com a adopção de um sistema informatizado de gestão da informação na saúde suplantam, aparentemente, os pontos negativos que se prendem, principalmente, com apoios e vontade profissional de mudar. Vários estudos realizados revelam que os maiores utilizadores dos sistemas de registo médico electrónico pertencem a escalões etários novos, e que a satisfação com estes sistemas é proporcional ao número de anos de uso. Ou seja, apesar de a adesão ser maior para os profissionais de saúde mais jovens, mais habituados às novas tecnologias, a verdade é que os utilizadores mais experientes revelam um elevado nível de satisfação. ${ }^{13}$ Este dado revela que, depois de ultrapassada a fase inicial de introdução de novos métodos, formação e adaptação, a rotina diária reajusta-se, podendo resultar em níveis de satisfação idênticos ou superiores e com parâmetros de qualidade acima dos anteriores.

Nos serviços nacionais de saúde, a iniciativa deve partir do poder político, fomentando uma vontade de apoiar todos os passos da implementação dos Sistemas e Tecnologias de Informação (Quadro III). ${ }^{6}$ Em Portugal, essa iniciativa aparece no programa do actual Governo Constitucional, existindo nele referências direc- 
QUADRO V. Objectivos ou o que melhorar na prescrição electrónica

- Motivar os médicos para a mudança

- Melhorar alertas em tempo real

- Integrar normas de orientação clínica

- Oferecer alternativas terapêuticas suportadas por evidência

- Actualizar regular e frequentemente as bases de dados

- Introduzir avisos sobre cuidados preventivos e/ou seguimento crónico

- Melhorar a comunicação com outras instituições (ex. farmácias)

- Desenvolver parcerias entre as várias empresas de forma a melhorar os suportes e minimizar as diferenças

tas à informatização dos registos e prescrição electrónica, como por exemplo «adoptar a prescrição electrónica de medicamentos e meios complementares de diagnóstico, com auxiliares de decisão clínica e informação sobre custos de dose média diária e sucedâneos», ${ }^{14}$ bem como no Plano Nacional de Saúde 2004-2010 que prevê a implementação de Sistemas Informáticos da Saúde. ${ }^{15}$ Quanto aos cuidados de saúde privados, a iniciativa parte da própria instituição, tendo como objectivo não só a melhoria da qualidade dos serviços prestados como a rentabilização dos recursos.

Interessa conseguir uma implementação massiva deste tipo de sistemas, uma vez que só com esta generalização se tornam mais fiáveis, mais atractivos e merecedores de confiança por parte dos clínicos. A presença de tecnologias e ferramentas poderosas ao dispor do médico é, só por si, um incentivo à sua utilização, por todos os benefícios já referidos para o médico de Medicina Geral e Familiar. ${ }^{16}$ Com a adaptação ao suporte informático como elemento integrado no consultório, este passará a ser mais um instrumento usado com naturalidade e aceite tanto pelo médico como pelo doente, cada vez mais consciente do seu direito a melhores cuidados de saúde, e até um factor integrador e de participação nos seus próprios cuidados.

Para conseguir o sucesso esperado, é também relevante o investimento na construção/evolução dos sistemas informatizados de registo em saúde, sendo necessário uma interacção constante e permanente com profissionais de saúde. Idealmente, estes sistemas devem ser compatíveis entre si, permitindo a sua interoperabilidade e devem ser actualizados regularmente e adaptados à gestão clínica. Esta procura de adequação obriga estes sistemas a disporem de suportes de apoio à decisão terapêutica: protocolos, normas de orientação clínica, alertas de toxicidade (interacções medicamentosas, interacções com problema de saúde, ajuste de dose, contra-indicações, duplicações).

Não obstante as aparentes vantagens do sistema informatizado, os profissionais não têm a certeza quanto aos reais benefícios do mesmo. A maioria acredita que os alertas só esporadicamente identificam eventuais interacções medicamentosas que não seriam perceptíveis ao clínico. Por outro lado, há uma noção de que a prescrição medicamentosa electrónica demora aproximadamente o mesmo que o habitual, sendo desmotivante para quem tenha a expectativa de vir a ganhar em tempo com a sua adopção. Há que avaliar e confirmar a existência (ou não) de melhores resultados, mais qualidade, mais eficiência e melhor gestão da consulta, conseguida a médio/longo prazo após adaptação às novas soluções. E porque não utilizar esta ferramenta dotada de rigor para avaliar vários parâmetros de qualidade, e premiar os profissionais com melhores índices?

Os estudos que avaliam o custo-benefício dos Sistemas e Tecnologias de Informação revelam um resultado claramente positivo, quer de poupança financeira, quer de diminuição dos erros de prescrição, poupança de tempo, melhores registos clínicos e até maior satisfação dos profissionais de saúde. Apesar de a evidência ainda não ser suficiente, este novo método de trabalho poderá trazer maior qualidade aos serviços de saúde prestados, nomeadamente para os doentes crónicos, melhores índices de produtividade e, eventualmente, melhores resultados quanto a índices de tratamento, de qualidade ou de satisfação dos doentes. Uma intervenção na motivação dos profissionais de saúde é dos factores mais relevantes a ter em conta na implementação da mudança.

\section{REFERÊNCIAS BIBLIOGRÁFICAS}

1. Showstack J, Lurie N, Larson EB, Rothman AA, Hassmiller S. Primary Care: The next renaissance. Ann Intern Med 2003; 138: 268-272.

2. Schade CP, Sullivan FM, Lusignan S. E-prescribing, efficiency, quality: lessons from the computerization of UK family practice. J Am Me Inform Assoc 2006; 13:470-5. 
3. Bell DS, Cretin S, Marken RS, Landman AB. A conceptual framework for evaluating outpatient electronic prescribing systems based on their functional capabilities. J Am Med Inform Assoc 2004; 11:60-70.

4. Wang SJ, Middleton B, Prosser LA, Bardon CG, Spurr CD, Carchidi PJ, et al. A cost-benefit analysis of electronic medical records in primary care. Am J Med 2003 Apr 1;114:397-403.

5. Pizzi LT, Suh DC, Barone J, Nash DB. Factors related to physicians'adoption of electrónica prescribing: results from a national survey.Am J Med Qual 2005;20:22-32.

6. Teich JM, Osheroff JA, Pifer EA, Sittic DF, Jenders RA. Clinical decision support in electronic prescribing: recommendations and an action plan. J Am Med Inform Assoc 2005; 12:365-76.

7. Robyn Tamblyn, Allen Huang, Robert Perreault, André Jacques, Denis Roy, James Hanley, Peter McLeod, Réjean Laprise. The medical office of the 21st century (MOXXI): effectiveness of computerized decision-making support in reducing inappropriate prescribing in primary care. CMA 2003;169(6):549-56. [cited 2008 Aug 16] Available from http://www. cmaj.ca/cgi/content/full/169/6/549

8. Dufour JC, Fieschi D, Fieschi M. Coupling computer-interpretable guidelines with a drug-database through a web-based system - The PRESGUID project BMC Medical Informatics and Decision Making 2004;4:112 [cited 2008 Jul 2] Available from http://www.biomedcentral.com/content/pdf/1472-6947-4-2.pdf

9. Terry AL, Thorpe CF, Giles G, Brown JB, Harris SB, Reid GJ, Thind A, Stewart M. Implementing electronic health records: Key factors in primary care Can Fam Physician. 2008;54:730-6 [cited 2008 Aug 14] Available from: http://www.cfp.ca/cgi/reprint/54/5/730?maxtoshow= \&HITS $=10$ \&hits $=10 \&$ RESULTFORMAT $=$ \&andorexactfulltext $=$ and \& searchid $=1 \& \mathrm{FIRSTINDEX}=0 \&$ sortspec $=$ relevance $\&$ volume $=54 \&$ firstpage $=730 \&$ resourcetype $=$ HWCIT

10. Fischer MA, Vogeli C, Stedman MR, Ferris TG, Weissman JS. Uptake of electronic prescribing in community-based practices. J Gen Intern Med;
23(4):358-63.

11. DesRoches CM, Campbell EG, Rao SR, Donelan K, Ferris TG, Jha A, Kaushal R, Levy DE, Rosenbaum S. Electronic Health Records in Ambulatory Care - A National Survey of Physicians. N Engl J Med. 2008;359:5060 [cited 2008 Jul 2] Available from: http://content.nejm.org/cgi/reprint/359/1/50.pdf

12. Taylor LK, Kawasumi Y, Bartlett G, Tamblyn R. Inappropriate Prescribing Practices: The Challenge and Opportunity for Patient Safety Healthcare Quarterly, 8(Sp) 2005: 81-85 [cited 2008 Aug 16] Available from http://www.longwoods.com/product.php?productid=17669\& cat= 399\&page $=1$

13. Tamblyn R, Huang A, Kawasumi Y, Bartlett G, Grad R, Jacques A, Dawes M, Abrahamowicz M, Perreault R, Taylor L, Winslade N, Poissant L, Pinsonneault A. The Development and Evaluation of an Integrated Electronic Prescribing and Drug Management System for Primary Care. J Am Med Inform Assoc. 2006;13:148-59.

14. Programa do XVII Governo Constitucional http://www.portugal. gov.pt/NR/rdonlyres/631A5B3F-5470-4AD7-AE0F-D8324A3 AF401/0/ProgramaGovernoXVII.pdf

15. DGS. Plano nacional de saúde 2004-2010: mais saúde para todos; Volume II - Orientações estratégicas. Lisboa: DGS; 2004.

16. Grossman JM, Gerland A, Reed MC, Fahlman C. Physicians' experiences using commercial e-prescribing systems. Health Affairs 2007 Apr 3;26(3):w393-w404

\section{ENDEREÇO DE CORRESPONDÊNCIA \\ Paula Broeiro \\ E-mail: paulabroeiro@iol.pt}

\section{AGRADECIMENTOS}

Agradecemos a preciosa colaboração dos colegas Daniel Pinto, Nuno Sousa e José Luís Biscaia.

\begin{abstract}
The electronic prescription happened as a need of the continuing improvement of the health care services and to manage limited resources. Electronic health records are always in evolution. United Kingdom is the best example with $100 \%$ users of electronic records in primary heath care. In the opposite, United States has $15 \%$ of users. Actually in Portugal the implementation of electronic health records begins its generalization, but evaluation data is insufficient.

Three electronic systems are operating in Portugal; they are designed as SAM, MedicineOne and VITACARE. All systems enable the prescription by International Common Denomination and by brand name. Differences about administrative efficiency and cost management of drugs are not substantive. The attractiveness of the evolutionary systems, like VITACARE, is to be centred in the patient including a teamwork philosophy. In the MedicineOne the prescription support was designed to aid the clinician with effectively functional and clinically relevant alerts on toxicity, interactions (drugs, disease, age, allergies). The system SAM, albeit more basic and with some limitations, is better than manual prescription.

The advantages with the adoption of the electronic prescription supports for the management of health information are greater than disadvantages, mainly due with lack of informatics support and the change of behaviours it implies. The most attractive aspects of the electronic prescription are: efficiency, usefulness, information content, user-friendly interface and Clinical Practice Guidelines integration into the clinician workflow processes prior to delivering recommendations on time, at the point of care.
\end{abstract}

Keywords: Electronic Prescription; Automated Prescription; Computerized Prescription Efficiency. 